\title{
Editorial on Wireless Networking Technologies for Smart Cities
}

\author{
Jaime Lloret $(\mathbb{D}),{ }^{1}$ Syed Hassan Ahmed $(\mathbb{D})^{2}$ Danda B. Rawat $(\mathbb{D})^{3}$ \\ Waleed Ejaz iD, ${ }^{4}$ and Wei Yu iD ${ }^{5}$ \\ ${ }^{1}$ Universitat Politecnica de Valencia, Valencia, Spain \\ ${ }^{2}$ Georgia Southern University, USA \\ ${ }^{3}$ Howard University, Washington, USA \\ ${ }^{4}$ Ryerson University, Toronto, Canada \\ ${ }^{5}$ Towson University, Maryland, USA \\ Correspondence should be addressed to Jaime Lloret; jlloret@dcom.upv.es
}

Received 7 August 2018; Accepted 7 August 2018; Published 1 October 2018

Copyright $\odot 2018$ Jaime Lloret et al. This is an open access article distributed under the Creative Commons Attribution License, which permits unrestricted use, distribution, and reproduction in any medium, provided the original work is properly cited.

Smart cities are becoming reality, due to the enormous research into the technology enablers and development of the Internet of Things (IoT) enabling a multitude of applications, which are built around various types of sensors. To manage the increased population of cities, there is a need to have more sustainable, environmentally-, and economicallyfriendly smarter cities and technologies. Moreover, the number of wireless technologies available for smart cities is increasing hugely [1]. Recently, despite the IoT devices constraints, such as their energy consumption [2], a number of smart devices appeared on a large scale, such as smart beds for monitoring and health data gathering during sleeping, like the SleepNumber smart bed, the Kolibree smart toothbrush, and the Belkin smart saucepan. These are few, with more applications and products to be showcased in the recent future.

The growing footprint of ultra-high-speed broadband networks, pervasive wireless networks, cloud computing, crowd sensing, and software-defined infrastructure connect smart/mobile devices to generate relevant city data on a massive scale. These advances will enable transformative applications and services that will enhance the quality of life while addressing important priorities such as real-time tracking, security, authenticity, and availability of classified information to the decision makers. Moreover, it will improve e-health services [3]. Similarly, to make a smart city, a strong communications infrastructure is required for connecting smart objects, people, and sensors. For instance, audio and video sensors support a variety of safety (monitoring) and other applications. Communication within cities involves multiple aggregations and access networks that can be either public or private. A city may gather data from smart devices and sensors embedded in the roadways, power grids, buildings, and other assets. It shares that data via a smart communications system that is typically a combination of wired and wireless networks, and in some cases mobile networks like Vehicular Ad Hoc Networks (VANETs) [4]. It then uses smart software to create valuable information and digitally enhanced services such as health care assistance, security and safety, real-time traffic monitoring, and managing the environment. The special issue contains 18 papers; each paper covers the subject from different prospective and thus offers readers a holistic view of different wireless technologies for smart cities.

Wireless sensor networks (WSNs) and IoT are key for the success of smart cities. In a paper entitled "Wireless Networking Performance in IoT Using Adaptive Contention Window", R. M. Bhavadharini et al. proposed an adaptive contention window to reduce the MAC overhead and retransmissions by determining active queue size at the contending nodes and the energy level of the nodes to improve TCP Performance. A novel indoor positioning system that considers the nonlinear discriminative feature extraction of received signal strength (RSS) using kernel local Fisher discriminant analysis (KLFDA) has been proposed by S. Imran and Y.-B. Ko in a paper entitled "A Novel Indoor 
Positioning System Using Kernel Local Discriminant Analysis in Internet-of-Things". The discriminating information in the RSS is reorganized and maximally extracted using KLDFA. In an article by M. Xie et al. entitled "Weight-Aware Sensor Deployment in Wireless Sensor Networks for Smart Cities", a reliability model of sensing area is established based on the sensing accuracy of the sensor. The sensing area is divided into sensing grids and different weights are allocated for those grids. An optimization problem is also formulated to maximize the trust degree of the WSNs for the efficient deployment of sensors.

Cellular networks are of paramount importance for the smart cities. In an article by S. Tahira et al. entitled "Handover Based IMS Registration Scheme for Next Generation Mobile Networks", a lightweight fast IMS mobility registration scheme is proposed to avoid unnecessary conventional registration phases such as security associations, authentication, and authorization. In an article entitled "A Reinforcement Learning Approach to Access Management in Wireless Cellular Networks" J. Moon and Y. Lim proposed the use of access class barring (ACB) to restrict the number of access attempts allowed in data transmission by utilizing strategic parameters and modeled the problem of determining the strategic parameters with a reinforcement-learning algorithm.

Cognitive radio networks can address spectrum scarcity issues in smart cities. In an article by A. Kheaksong et al. entitled "Multicriteria Parent Selection Using Cognitive Radio for RPL in Smart Grid Network", a parent selecting mechanism is proposed to improve workload balancing and lifetime differences of all meters. The proposed mechanism is based on three criteria including expected transmission count, residual energy, and expected transmission time. In an article entitled "Adaptive and Blind Wideband Spectrum Sensing Scheme Using Singular Value Decomposition" Z. Hu et al. proposed an adaptive and blind reduced multiple measurement vectors boost (ABRMB) scheme based on singular value decomposition for wideband spectrum sensing.

Energy-efficient protocols and algorithms are also of prime importance for smart cities. In article entitled "EEMRP: Energy-Efficient Multistage Routing Protocol for Wireless Sensor Networks" M. K. Khan et al. proposed an energy-efficient routing protocol for wireless sensor networks. The proposed protocol consists of a routing algorithm for the transmission of data, cluster head selection algorithm, and a scheme for the formation of clusters. An energy-efficient unequal chain length clustering (EEUCLC) protocol is proposed by M. Baniata and J. Hong in a paper entitled "Energy-Efficient Unequal Chain Length Clustering for Wireless Sensor Networks in Smart Cities". The protocol consists of a suboptimal multihop routing algorithm to reduce the burden on the cluster head and a probabilitybased cluster head selection algorithm to prolong the network lifetime.

Smart cities also have to deal with a Big Data for performance enhancement and decision-making. A smart city framework based on Big Data analytics is proposed by B. N. Silva et al. in an article entitled "Big Data Analytics Embedded Smart City Architecture for Performance Enhancement through Real-Time Data Processing and Decision-Making”. The proposed framework operates on (i) data generation and acquisition, (ii) data management and processing, and (iii) application level initiates execution of the events. M. M. Iqbal et al. in an article entitled "Augmenting HighPerformance Mobile Cloud Computations for Big Data in AMBER" proposed an innovative execution of assisted model building with energy refinement (AMBER) with force field empirical formula, using message passing interface (MPI) infrastructure on high-performance mobile cloud computing. The main objective of this work is facilitating research community with a mobile cloud of laptops capable of doing the heavy processing.

Security and privacy in smart cities have also been addressed in recent years. In an article entitled "TrustyFeer: A Subjective Logic Trust Model for Smart City Peer-toPeer Federated Clouds" by H. Kurdi et al. proposed a trust management system called TrustyFeer that allows peers to evaluate the trustworthiness of other peers based on subjective logic opinions, formulated using peers' reputations and service level agreements (SLAs). A framework for performing a novel, template-based fuzzing technique on the message queue telemetry transport is proposed by S. H. Ramos et al. in an article entitled "MQTT Security: A Novel Fuzzing Approach". An agent-based open-source framework for testing different policies for normalizing and controlling self-reported priorities, with its simulator called ABS-smartPriority is proposed by I. García-Magariño and R.1 Lacuesta in an article entitled "ABS-SmartPriority: An Agent-Based Simulator of Strategies for Managing SelfReported Priorities in Smart Cities". This simulator is illustrated by two different strategies, i.e., smart policy for tracking this history of citizens and control mechanism for the priorities self-reported by the users. In order to reduce computation cost and achieve better security, in "Lightweight Data Aggregation Scheme against Internal Attackers in Smart Grid Using Elliptic Curve Cryptography” D. He et al. proposed a lightweight data aggregation scheme against internal attackers in the smart grid environment using elliptic curve cryptography.

The last group of articles in this special issue focus on wireless technologies for different applications in smart cities. An approach for mass-customization with the integration of smart retail and smart production is proposed by $\mathrm{M}$. Karaköse and H. Yetiş in an article entitled "A Cyberphysical System Based Mass-Customization Approach with Integration of Industry 4.0 and Smart City". The objective is to reduce the waiting time of customers. A distributed groupbased mobility management scheme is proposed by M. Gohar et al. in a paper entitled "Distributed Group-Based Mobility Management Scheme in Wireless Body Area Networks". A local mobility anchor (LMA) function is implemented by each mobile access gateway (MAG) and the handover operation is performed between two neighbouring MAGs without the help of LMA. In "Semantic Interoperability in Heterogeneous IoT Infrastructure for Healthcare" S. Jabbar et al. proposed an IoT based semantic interoperability model (IoT-SIM) to provide semantic interoperability among heterogeneous IoT devices in health care domain. 
All of the papers in special issue highlight the importance of wireless technologies for the smart cities. In addition, authors investigated the issues related to security and privacy and Big Data analysis in smart cities. In a nutshell, the integration of new wireless technologies with the evolved current ones can bring tremendous improvement in future smart cities.

\section{Conflicts of Interest}

The authors declare that there are no conflicts of interest regarding the publication of this article.

\section{Acknowledgments}

We also would like to thank editorial staff, editor in chief, contributing authors, reviewers, and production staff for bringing our special issue to the level of success.

Jaime Lloret Syed Hassan Ahmed

Danda B. Rawat Waleed Ejaz

Wei Yu

\section{References}

[1] L. Garcia, J. M. Jiménez, M. Taha, and J. Lloret, "Wireless Technologies for IoT in Smart Cities," Network Protocols and Algorithms, vol. 10, no. 1, pp. 23-64, 2018.

[2] S. Rani, R. Talwar, J. Malhotra, S. H. Ahmed, M. Sarkar, and H. Song, "A novel scheme for an energy efficient internet of things based on wireless sensor networks," Sensors, vol. 15, no. 11, pp. 28603-28626, 2015.

[3] A. Rghioui, S. Sendra, J. Lloret, and A. Oumnad, "Internet of Things for Measuring Human Activities in Ambient Assisted Living and e-Health," Network Protocols and Algorithms, vol. 8, no. 3, pp. 15-28, 2016.

[4] Y. He, Z. Wei, G. Du, J. Li, N. Zhao, and H. Yin, "Securing cognitive radio vehicular Ad hoc networks with fog computing," Ad Hoc \& Sensor Wireless Networks, vol. 40, no. 1-2, pp. 73-95, 2018. 


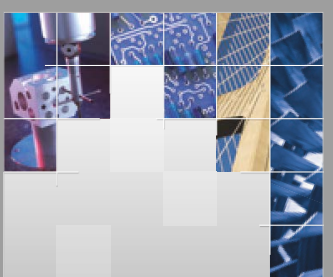

\section{Enfincering}
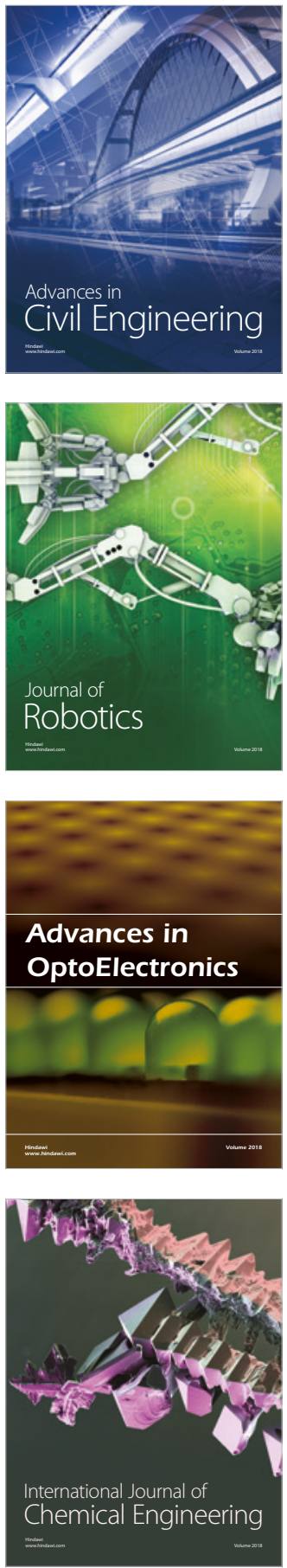

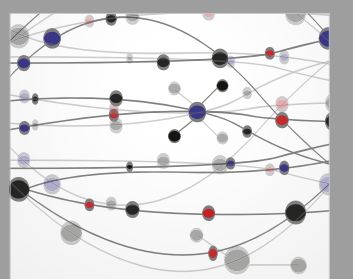

\section{Rotating \\ Machinery}

The Scientific World Journal

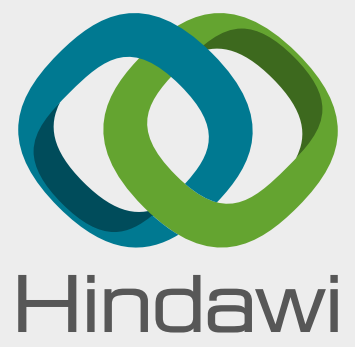

Submit your manuscripts at

www.hindawi.com
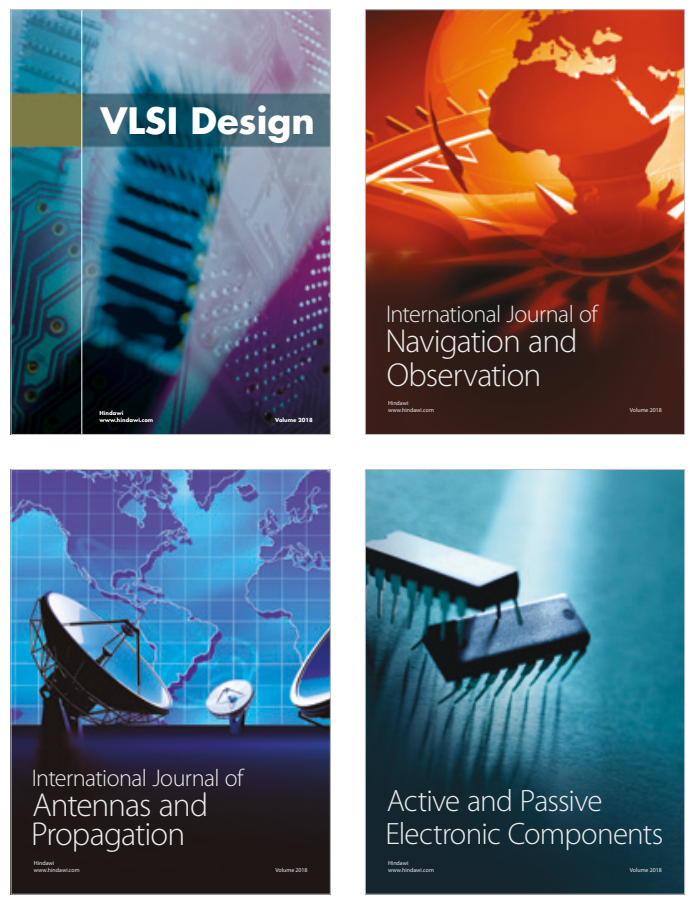
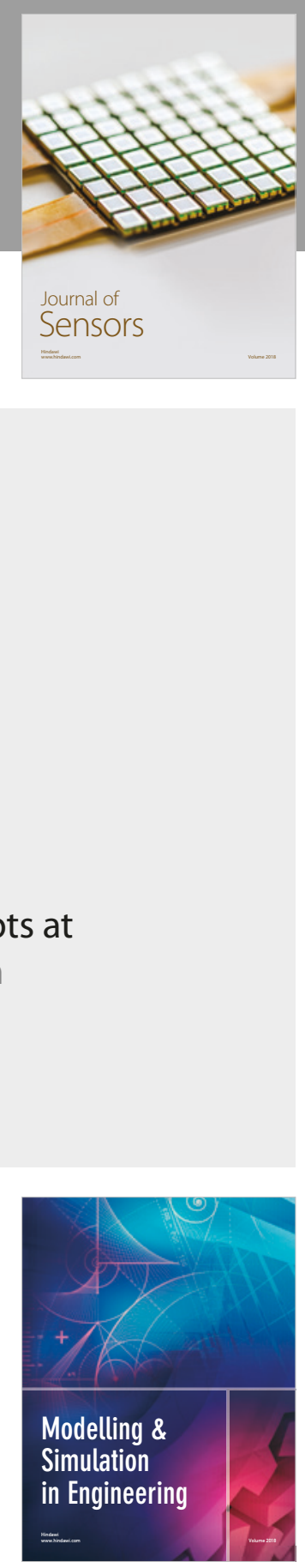

\section{Advances \\ Multimedia}
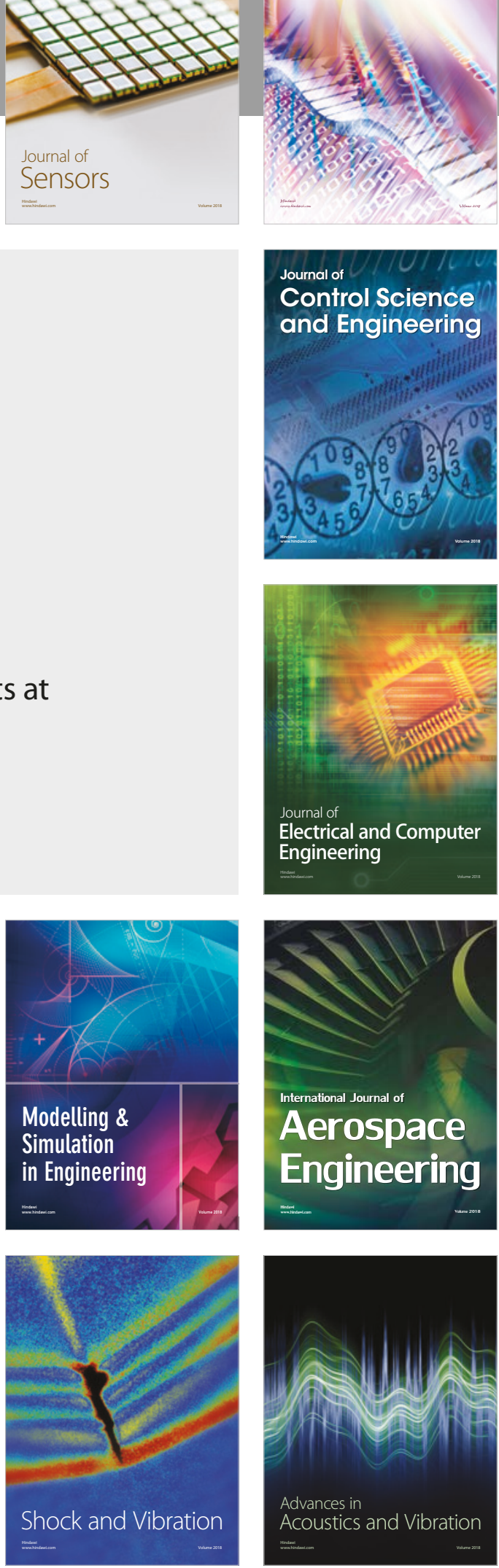